\title{
Luminescence Detection of Latent Fingermarks on Non-porous Surfaces with Heavy-Metal-Free Quantum Dots
}

\author{
Sorour Shahbazi, ${ }^{[a]}$ Rhiannon Boseley, ${ }^{[a]}$ Braden Grant, ${ }^{[a]}$ Dechao Chen, ${ }^{\text {[a] }}$ Thomas Becker, ${ }^{[a]}$ \\ Oluwasesan Adegoke, ${ }^{[b]}$ Niamh Nic Daéid, ${ }^{[b]}$ Guohua Jia, ${ }^{*[a]}$ Simon W. Lewis ${ }^{*[a]}$ \\ [a] S. Shahbazi, R. Boseley, B. Grant, D. Chen, Dr T. Becker, Dr G. Jia, Prof S.W. Lewis \\ School of Molecular and Life Sciences \& Curtin Institute of Functional Molecules and Interfaces \\ Curtin University \\ GPO Box U1987, Perth, WA, 6845, Australia
}

[b] O. Adegoke, Prof N. Nic Daéid

Leverhulme Research Centre for Forensic Science, School of Science and Engineering

University of Dundee

Dundee, Scotland, UK

*Corresponding authors E-mail: S.lewis@curtin.edu.au; guohua.jia@curtin.edu.au

\begin{abstract}
Current and proposed nanoparticle-based techniques for development of latent fingermarks suffer a number of drawbacks such as complicated, multi-step and time-consuming procedures, batch-to-batch variability, expensive reagents, large background noise and toxicity. Here, we introduce a promising green development technique based on heavymetal-free quantum dots for the detection of latent fingermarks on nonporous surfaces. Red-near infrared luminescent $\mathrm{Cu}-\mathrm{In}-\mathrm{S} / \mathrm{ZnS}$ core-shell QDs solution was produced in large scales using a water-based, simple and fast method using $\mathrm{N}$-acetylcysteine as a biocompatible surfactant to coat the particles. The coated QDs were applied to the successful development of latent fingermarks deposited on a variety of surfaces, including highly patterned polymer banknotes and the sticky side of adhesive tape.
\end{abstract}

\subsection{Introduction}

The detection of latent (not visible to the naked eye) fingermarks has been carried out for more than a century to enable associations between individuals and objects found at crime scenes to be evaluated $[1,2]$. In more recent years innovative methods and materials based on nanotechnology have been applied to the visualisation of latent fingermarks with noticeable progress from specifically targeting fingermark components [3-5].

Luminescent quantum dots (QDs) manifesting large cross sections of the UV/visible spectral range, narrow and size-dependent emission wavelengths, high quantum efficiencies, and enhanced photostabilities, have been applied to the development of latent fingermarks on non porous surfaces over the last two decades [6, 7]. However even though cadmium-based QDs such as CdS [6], CdSe [8] and CdTe $[9,10]$ have successfully visualised latent fingermarks, issues relating to the toxicity associated with heavy metals remain a concern [11]. In order to increase their biocompatibility some reported methods have included covering the QDs with a surfactant or over coating them with a non-toxic inorganic shell [12], however, even with these techniques, the underlying issue of toxicity is unsolved. Heavy-metal-free QDs such as carbon [13], $\mathrm{Cu}$ - and $\mathrm{Mn}$-doped $\mathrm{ZnS}[14,15]$ quantum dots have been proposed as alternatives, however, the latent fingermarks visualised by these QDs, which luminesce in the visible part of the spectrum, exhibit reduced sensitivity of the detection due to background noise [13-15].

Luminescence increases the contrast between latent fingermark ridges and the underlying substrate by moving the means of detection outside of the ambient illumination [16, 17]. Materials luminescing at longer wavelengths, (red to near infrared (NIR) region), have the potential to improve detectability of fingermarks since there is less interference in this region. However, only a limited number of NIR-based techniques have been reported for the detection of fingermarks and these techniques suffered drawbacks including toxicity, being a post treatment rather than a standalone process and lack of contrast [18-20].

To fill these technology gaps, three main objectives for this work were identified: first, preparation of a red-NIR luminescent heavymetal-free QDs water-based solution; second, modification of the QDs to boost their affinity to interact with the fingermark residue and third, making the procedure simple and practical for operational use.

Ternary metal complex chalcogenide, Cu-In-S/ZnS (CIS-ZS) coreshell quantum dots are of low toxicity and exhibit strong and broad absorption of ultraviolet and visible light, intense photoemission and reasonable stability under photoexcitation [21, 22]. CIS-ZS QDS have been used in a variety of light-emitting applications such as solar cells [23], electro-luminosity [24], photocatalysts [25] and bioimaging [26-28]. However, to the best of our knowledge, ternary core-shell QDs with photoluminescence (PL) in red/NIR range have not been used for fingermark visualisation. This study provides a proof of concept for the use of CIS-ZS quantum dots in aqueous solution, modified with surfactant coatings, for the detection of latent fingermarks on a range of non-porous surfaces.

\subsection{Materials and Methods}

\subsection{Chemicals}

Copper acetate $(\mathrm{Cu}(\mathrm{Ac}) 2,99.99 \%)$, indium acetate (In(Ac)3, 99.99\%), zinc acetate $(\operatorname{Zn}(\mathrm{Ac}) 2,99.99 \%)$, 1-dodecanethiol (DDT, 98\%), 1octadecene (ODE, 90\%), oleic acid (OA, 90\%), oleylamine (OAm, 97\%), 11-Mercaptoundecanoic acid (MUA, 98\%), potassium hydroxide pellets (>85.0\%)3-mercaptopropionic acid (MPA, $\geq 99 \%$ ), copper (II) chloride dihydrate ( $\geq 99.99 \%)$, ammonia solution (30\%), indium (III) chloride (99.99\%), sodium sulfide, zinc acetate dihydrate ( $\geq 99 \%$ ) and $\mathrm{N}$-acetyl-cysteine (NAC, $\geq 99 \%$ ) were purchased from Sigma-Aldrich. All chemicals were used without further purification.

\subsection{Nanoparticle Synthesis}

CIS-ZS core-shell QDs synthesised in organic solvent: The Zn stock solution was prepared before reaction by dissolving $0.367 \mathrm{~g}$ of $\mathrm{Zn}(\mathrm{Ac})_{2}(2 \mathrm{mmol})$ in $5 \mathrm{ml}$ of ODE/OAm (4:1 volume ratio) at $160^{\circ} \mathrm{C}$ under $\mathrm{N}_{2}$ flow. Colloidal CIS core QDs were synthesised based on a 
literature method.[29] $0.0584 \mathrm{~g}$ of $\ln (\mathrm{Ac}) 3(0.2 \mathrm{mmol})$ and $0.0363 \mathrm{~g}$ of $\mathrm{Cu}(\mathrm{Ac}) 2(0.2 \mathrm{mmol})$ were added into a $50 \mathrm{ml}$ three-neck flask with $2.0 \mathrm{~mL}$ of DDT, $3 \mathrm{~mL}$ of ODE and $0.3 \mathrm{~mL}$ of OA. The reaction mixture was heated to $100{ }^{\circ} \mathrm{C}$ for 10 min under $\mathrm{N} 2$ gas flow to form a clear solution. Then, the temperature was raised to $260^{\circ} \mathrm{C}$ in $3 \mathrm{~min}$. The CIS QDs were allowed to grow. The colour of the solution changed from slightly yellow to yellow, red, and finally black. Once the solution turned black, indicating the bigger core of CIS QDs, the temperature was dropped to $100{ }^{\circ} \mathrm{C}$. For $\mathrm{ZnS}$ shell growth, the synthesised CIS QD crude solution was directly used without purification. In a typical synthesis, the $\mathrm{Zn}$ stock solution was injected into the reaction solution. The temperature was raised to $230^{\circ} \mathrm{C}$ and kept for $30 \mathrm{~min}$. Then the solution was quenched. The particles were precipitated and washed by hexane/ethanol. The obtained CIS-ZS QDs were redispersed in chloroform. Ligand exchange was carried out by following a previously reported method [30], $0.5 \mathrm{~g}$ of MUA in $7 \mathrm{ml}$ of chloroform was added to the $2 \mathrm{ml}$ of CIS-ZS core-shell QDs solution. $5 \mathrm{ml}$ of $0.1 \mathrm{M} \mathrm{KOH}$ solution was added and stirred overnight to let the QDs transfer from organic solution to water. Then the aqueous phase including the QDs was separated using a pipette and centrifuged at $6000 \mathrm{rpm}$ for 2 min. Supernatant was decanted and the QDS were re-dispersed in water for further use.

Water-synthesis of CIS-ZS core-shell QDs: In a typical synthesis of the QDs, a mixture of $95 \mathrm{~mL}$ of deionised water, $2 \mathrm{~mL}$ of MPA (1.0 M), $1 \mathrm{~mL}$ of $\mathrm{CuCl}_{2}(0.1 \mathrm{M}), 0.4 \mathrm{~mL}$ of $\mathrm{NH}_{4} \mathrm{OH}(30 \%), 0.5 \mathrm{~mL}$ of $\mathrm{InCl}_{3}$ $(1.0 \mathrm{M})$ and $1 \mathrm{~mL}$ of $\mathrm{Na}_{2} \mathrm{~S}(1.0 \mathrm{M})$ aqueous solutions were heated to 90-95 ${ }^{\circ} \mathrm{C}$ while aggressively stirred in a flask for $20 \mathrm{~min}$. Then different amounts of $\mathrm{Zn}(\mathrm{Ac})_{2}(1.0 \mathrm{M})$ were added dropwise. The mixture was stirred for another $15 \mathrm{~min}$ and then left to cool down to the room temperature. In a typical post-synthesis treatment, where applicable, $0.3 \mathrm{ml}$ MPA or $1.08 \mathrm{~g}$ of NAC was added to the as-synthesised solution.

\subsection{Transmission electron microscopy}

Transmission electron microscopy (TEM) was performed on a JEOL 2100 transmission electron microscope with a tungsten filament running at an accelerating voltage of $120 \mathrm{keV}$. TEM specimens were prepared by depositing one drop of the QDs solution on carboncoated copper grids.

\subsection{Optical spectroscopy}

A PerkinElmer Lambda 35 ultraviolet-visible (UV-Vis) absorption spectrophotometer with a wavelength range of $190-1000 \mathrm{~nm}( \pm 0.1$ $\mathrm{nm}$ wavelength accuracy) was used to collect optical density of the solutions. The instrument was equipped with deuterium and tungsten halogen lamps and a Seya Namokia monochromator. PerkinElmer UV WinLab software (version 6.0.2.0723) was used for analysing the data. Luminescent characteristics of the prepared QDs were investigated using a Cary Eclipse fluorescence spectrophotometer equipped with two Czerny-Turner monochromators and a $15 \mathrm{~W}$ Xenon Flash Lamp, Schwartzchild collection optics and two photomultiplier detection tubes. The spectral range of this spectrometer is from the deep ultraviolet (UV) at $190 \mathrm{~nm}$ to near infrared (NIR) at $900 \mathrm{~nm}$ with an accuracy of $\pm 1.0 \mathrm{~nm}$. The data were analysed with Cary Eclipse Scan Application software (version 1.2).

\subsection{Collection and treatment of latent fingermarks}

Substrates including glass slide, new \$5 Australian polymer banknote and aluminium drink can were washed with detergent and deionised water and other substrates including black adhesive insulating tape, transparent sticky tape, aluminium foil and zip-lock bag were used in their new state. Donors both male and female aged between 20-50 years were asked not to wash their hands for at least half an hour before fingermark collection. For collecting uncharged (natural) latent fingermarks, donors only rubbed their hands together and touched the substrates for approximately 5 seconds. For collecting charged latent fingermarks, donors were asked to first rub their hands on their face and hair and then rub them together. Fingermarks were kept in an office environment away from sunlight without any light protection. Fresh fingermarks were treated immediately after collection. The temperature and the relative humidity were monitored using a Digitech QP-6013 datalogger over the period of storage, producing recorded temperature and relative humidity ranges of $20-23{ }^{\circ} \mathrm{C}$ and $25-70 \%$ $\mathrm{RH}$, respectively.

The collected latent fingermarks were immersed in the QDs working solutions. The working solutions were orbitally shaken on a PathTech Basic Orbital Mixer for different duration. The developed fingermarks were rinsed and shaken in a deionised water bath to remove excess QDs adhered to the substrate. Finally, the developed fingermarks were dried in an oven at $30^{\circ} \mathrm{C}$ for a maximum of $10 \mathrm{~min}$.

\subsection{Photography}

Following development, developed latent fingermarks were photographed in a dark room using a Nikon D300 camera with an AF-S Micro NIKKOR $60 \mathrm{~mm}$ lens, a Tamron $62 \mathrm{~mm}$ Skylight $1 \mathrm{~A}$ filter and an Anti-Glare Schott RG630 AG 1\%(nom) deep red filter (long-pass $613 \mathrm{~nm}$ ). The Nikon camera was connected to a desktop computer running Camera Control Pro 2 software (version 2.8.0) to use the live view and remote-control functions of the camera. The camera was mounted overhead on a Firenze mini repro stand in a darkened room. The photography specifications of the fingermarks photos were chosen to enhance contrast and visibility of the treated marks, and are specified in Table S1. Developed fingermarks on various substrates were illuminated with a forensic light source (Polilight PL500, Rofine, Australia). Photos were processed and adjusted in brightness using Adobe Photoshop CC (version 19.1.16) to make fingermarks details more visible. However, the same modifications were applied on the split fingermarks.

\subsection{Results and Discussion}

A preliminary investigation was carried out using CIS-ZS QDs synthesised in organic solvent which were then transferred to the aqueous phase by ligand exchange. The surfactant coating has a dual role in that it allows dispersion of the QDs in aqueous solution, as well as providing the capability of being attracted to the latent fingermark residue. The aqueous solution of QDs gave promising results demonstrating that is possible to develop fingermarks with CIS-ZS QDs on non-porous surfaces (see Figures S1 and S2 in ESI).

Synthesis of QDs in an organic phase produces uniform and brightly luminescent products. However, an organic synthesis method is complicated, time-consuming and expensive due to the tedious preparation steps, inert atmosphere condition, and high reaction temperature $\left(>200^{\circ} \mathrm{C}\right)[26,31]$. Also, the yield of QDs is very low ( $\sim$ tens of $\mathrm{mg}$ ) and the required organic substances raise significant safety issues [32, 33]. Furthermore, as a post-synthesis treatment, the QDs must be transferred from organic to aqueous phase by ligand exchange to render them biocompatible and applicable to 
fingermark development, which is a long process and may result in agglomeration and loss of luminescence [9]. We experienced all the aforementioned issues during the organic-synthesis and ligand exchange of the CIS-ZS QDs as well as their application to fingermarks development (Figure S1 and Figure S2). These drawbacks limit the usage of this method for application in an operational environment.

Preparation of QDs in water has the potential to be more rapid, simpler, more biocompatible and inexpensive. However, the surfaces of the as-prepared QDs have dangling bonds which are tentatively passivated by organic surfactants. Subsequent exposure of the synthesised QDs to an aqueous environment results in surface oxidation and a decrease in luminescence efficiency [31]. Previous studies have used nitrogen gas purging to attempt to solve this problem but this method cannot completely suppress the quenching effect $[14,32]$. A more effective strategy, namely passivation of QDs with an inorganic shell made of other semiconductor materials, noticeably minimises the trap-state defects and increases the luminescent quantum yield and stability $[34,35]$. Aqueous-synthesis of CIS-ZS QDS has been achieved using microwave-assisted [26] and hydrothermal methods [36], however these approaches suffer drawbacks including the necessity of special facilities and being a time consuming procedure. More recently, a convenient, fast and inexpensive water-based synthesis method with a possibility of upscaling has been established to produce CIS-ZS QDs in a safer way [37, 38]. In this approach, a mixture of copper, indium and sulfur precursors in water is heated at low temperatures $\left(<100^{\circ} \mathrm{C}\right)$ to produce the $\mathrm{Cu}-\mathrm{In}$-S core, and then a zinc precursor is added to form the shell around the cores. We used this approach with modifications to produce CIS-ZS working solutions that could be used directly for the effective visualisation of latent fingermarks on different non-porous substrates without any further changes such as purification or $\mathrm{pH}$ and temperature adjustments.

Colloidal CIS-ZS core-shell QDs were synthesised in water in a simple one-step technique. First, aqueous solutions of $\mathrm{CuCl}_{2}, \mathrm{InCl}_{3}$, $\mathrm{Na}_{2} \mathrm{~S}$ were mixed in water in presence of a surfactant and ammonium solution. The reaction mixture was heated and kept at $90-95^{\circ} \mathrm{C}$. Cu and In salts participate in a precipitation reaction with sodium sulfide and produce the $\mathrm{Cu}-\mathrm{In}$-S spherical nanoparticles [31] Zn (II) precursor was added to the reaction mixture without any purification and the ZnS layers epitaxially grew on the cores as a shell producing a luminescent CIS-ZS QDs solution (Figures 1a-1c). This QD solution can be prepared within 90 min on a large scale (at least $400 \mathrm{~mL}$ of $0.96 \mathrm{M}$ solution per batch). Figure $1 \mathrm{~d}$ and Figures S3a-S3d show the TEM images of the synthesised CIS-ZS core-shell QDs. The size of the QDs was less than $5 \mathrm{~nm}$. Absorption (Ab) and PL spectra of the CIS core and CIS-ZS core-shell QDs are illustrated in Figure 1e.

The synthesised QDs fluoresced at deep red to NIR (emission maximum at $660 \mathrm{~nm}$ ) when excited in a wide range of wavelengths from UV to red region. The growth of ZnS shells around the CIS cores noticeably increased the PL intensity (approximately 15 times). Increasing the amount of $\mathrm{Zn}^{2+}$ precursor resulted in significant enhancement of the luminosity due to an increase of the thickness of the shell (Inset in Figure 1e); however, it led to a slightly blue-shift from $680 \mathrm{~nm}$ for the core to $660 \mathrm{~nm}$ for the final product. This is most probably related to the surface reconstruction rather than the diffusion of $\mathrm{Zn}$ into the CIS core taking into account the relatively low temperature of $90-95^{\circ} \mathrm{C}[27]$.
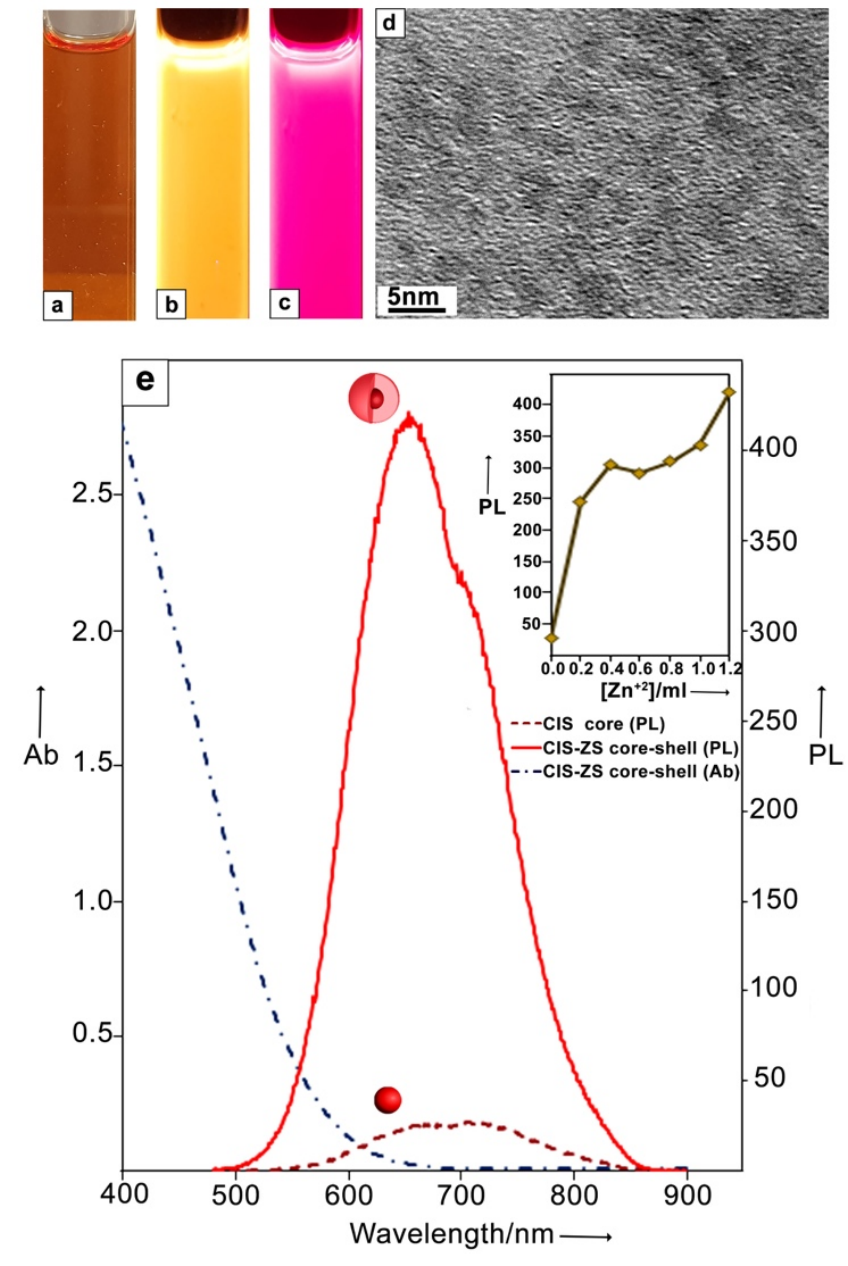

Figure 1. (a-c) CIS-ZS QDs solution photographed under (a) no illumination and (b and c) $350 \mathrm{~nm}$ illumination and through ( $\mathrm{a}$ and b) no filter and (c) deep red filter (longpass $613 \mathrm{~nm}$ ). (d) TEM image of CIS-ZS QDs. (e) PL (excited at $350 \mathrm{~nm}$ ) and Ab spectra of CIS core and CIS-ZS core-shell QDs. Inset in (e): maximum (excited at $350 \mathrm{~nm}$ ) PL of CIS-ZS QDs with different amounts of $\mathrm{Zn}^{2+}$ precursor ([Zn $\left.\left.n^{2+}\right]\right)$.

Aging the prepared QDs solution under light for about one month, noticeably boosted the PL intensity (Figure S3e) due to the photodegradation of the surfactant leading to thickening of the shell, increasing the crystallinity and reducing the surface defects, as reported previously $[32,39]$. Addition of more than $0.6 \mathrm{~mL}$ of $\mathrm{Zn}$ precursor led to the formation of a cloudy solution due to agglomeration and precipitation of the QDs (Figures S4). This unstable solution was undesirable as it hindered fingermark development, developing less ridge detail.

Initially 3-Mercaptopropionic acid (MPA) surfactant (the same surfactant used during the synthesis) was added to the prepared solution to stabilize the quantum dots as a post-synthesis step to prevent sedimentation by providing an electrostatic shield against inter-particle interactions. The long carbon chain and carboxylic group in the chemical structure of MPA was also proposed to increase both polar and non-polar interaction affinity with the components within the fingermark residue. Although only used in small amounts, MPA is a toxic and malodorous chemical. To increase the safety of the working solution alternative molecules were explored as capping ligands, including L-cysteine and $\mathrm{N}$ acetylcysteine (NAC), to replace MPA in the post-synthesis step. Both are non-hazardous chemicals with NAC used for medical 
purposes such treatment of paracetamol poisoning [40]. Xu et al. has previously demonstrated that NAC-capped QDs developed more ridge details of latent fingermarks compared to other ligands including MPA [15], fingermarks developed on transparent tape in our studies with NAC as the endcapping ligand consistently producing defined ridge detail and contrast when compared to MPA, with L-cysteine yielding no development of latent fingermarks (See Figure S5). This is suggested to be due to the variety of functional groups present in NAC, such as thiols, carbonyl and amide groups which enable interactions with lipids and amino acids present in fingermark secretions. Further studies are required to investigate the nature of the interaction of the quantum dots with the latent fingermark, as well as exploring other potential endcapping ligands.

A typical procedure for the treatment of latent fingermarks with CIS-ZS QDs solution involved immersing the sample in the solution and agitating for varying durations, with most substrates requiring no longer than 20 minutes. International Fingerprint Research Group (IFRG) guidelines relating to initial proof of concept (Phase 1) studies were followed to conduct the experiment [41]. A range of substrates including transparent, black and blue adhesive insulating tapes, glass slides, aluminium foil, aluminium drink cans and zip-lock bags were tested and developed fingermark ridge detail (see Figure 2). The sticky side of adhesive tapes demonstrated particularly promising results, Figure $2 \mathrm{~g}$ shows excellent ridge detail including the sweat pores of a natural latent fingermark on a transparent sticky tape developed with the NACcoated CIS-ZS core-shell QDs. To further investigate potential of the technique, the natural split fingermarks of 6 donors on transparent tape, aluminium foil and glass slides were developed. The results demonstrated full or partial development of the latent fingermarks from all 6 donors (Figure S6).

As a proof of concept, charged latent fingermarks on new Australian polymer banknotes as a nonporous multi-coloured substrate were treated with MPA capped CIS-ZS QDs, development produced marks well enough to distinguish high levels of ridge detail (Figure $3 \mathrm{~b}, \mathrm{~d}$ ).
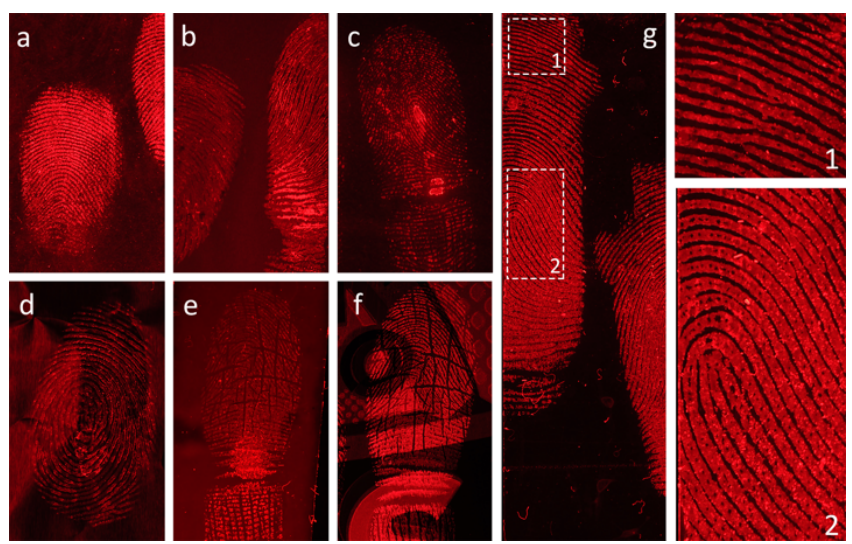

Figure 2. Fresh developed $(a-d, g)$ natural and $(e, f)$ charged latent fingermarks on (a) glass slide, b) black adhesive insulating tape, (c) zip lock bag, (d) aluminium foil, (e) blue adhesive insulating tape, (f) aluminium drink can and (g) transparent adhesive tape. The marks were illuminated at $350 \mathrm{~nm}$ and pictured through the deep red filter. The marks were shaken at ((a-d and f-g) $20 \mathrm{rpm}$ for (b)10 min, (a,c) $20 \mathrm{~min},(\mathrm{~d}) 15 \mathrm{~min}$ (f) $2 \mathrm{hrs}$ and (g) $5 \mathrm{~min}$ in CIS-ZS QDs solution, (e) the mark was treated with no shaking for $20 \mathrm{~min}$. The marks were illuminated at $350 \mathrm{~nm}$ and pictured through the deep red filter. See Table S1 for the photography conditions.
Being luminescent in deep red to NIR region made it possible to visualise the latent fingermarks on these multi-coloured and multipatterned substrates. The CIZ-Zn QDs can be excited with a broad range of wavelengths. This is advantageous when working with inherently luminescent surfaces. Figure $3 f$ shows that the security feature of a UV-fluorescent bird interfered with the fingermark on top of it. To visualise the hidden part of the fingermark, it was illuminated at $450 \mathrm{~nm}$ instead of $350 \mathrm{~nm}$ leading to the elimination of fluorescence from the bird feature (Figure $3 \mathrm{~g}$ ).
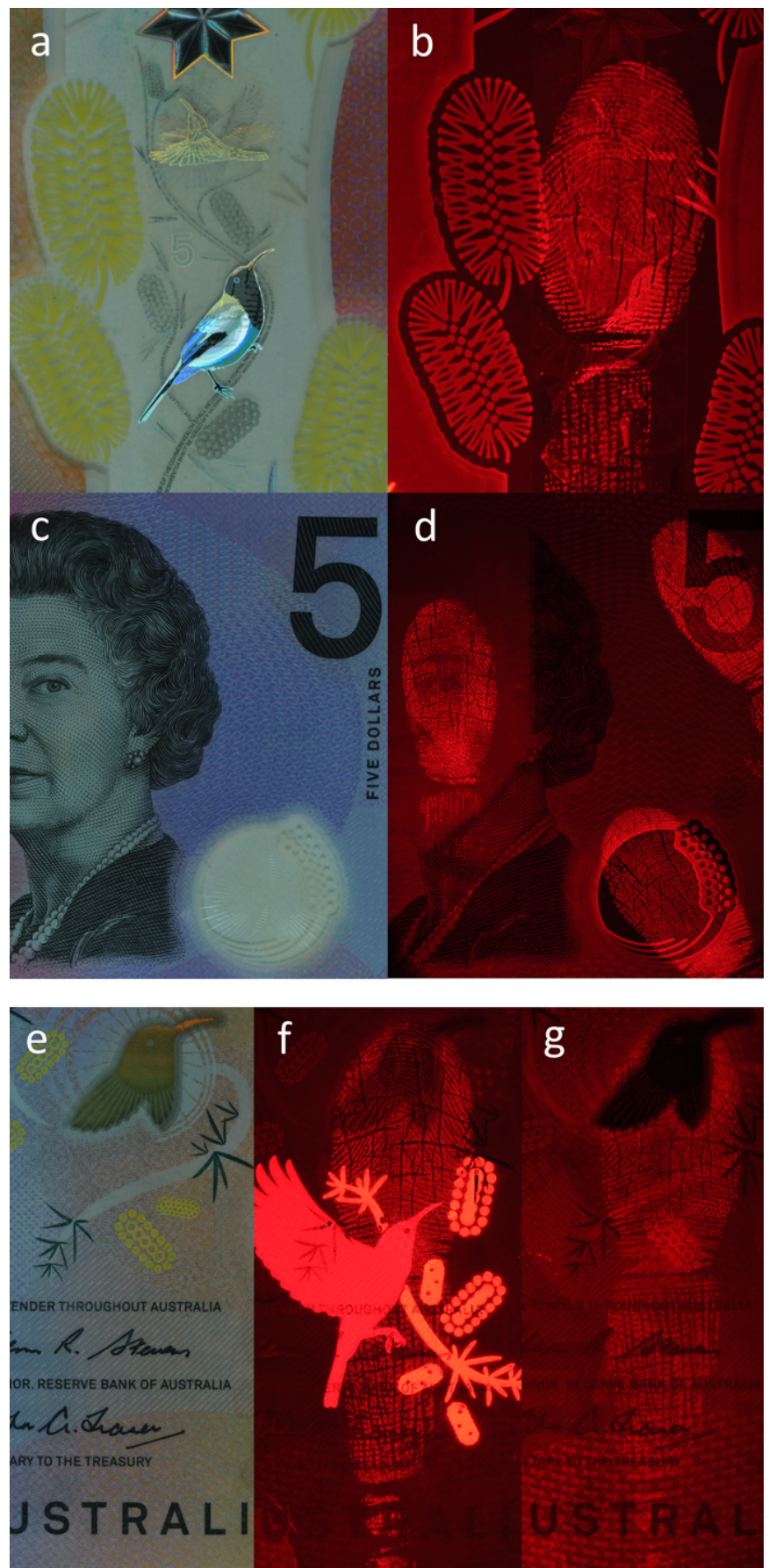

Figure 3. (a,c,e) Unilluminated and (b,d,f) illuminated at $350 \mathrm{~nm}$ and (g) $450 \mathrm{~nm}$ charged latent fingermarks (one female donor) deposited on Australian polymer banknote. The illuminated marks were pictured through a deep red filter. The marks were shaken at $20 \mathrm{rpm}$ for $20 \mathrm{~min}$ in a MPA-coated CIS-ZS QDs solution. See ESI ${ }^{\dagger}$ and Table S1 for the photography details. 
Subsequent testing explored natural fingermarks on these substrates with NAC capped CIS-ZS QDs, Figure 4 demonstrates the level of ridge detail developed from 8 day old natural fingermarks. Whilst a larger number of donors showed poor development, the use of aged, natural marks gives a more realistic representation of fingermark evidence, further investigation will continue to explore the use of CIS-ZS QD's on these surfaces, particularly on the role of the surfactant.
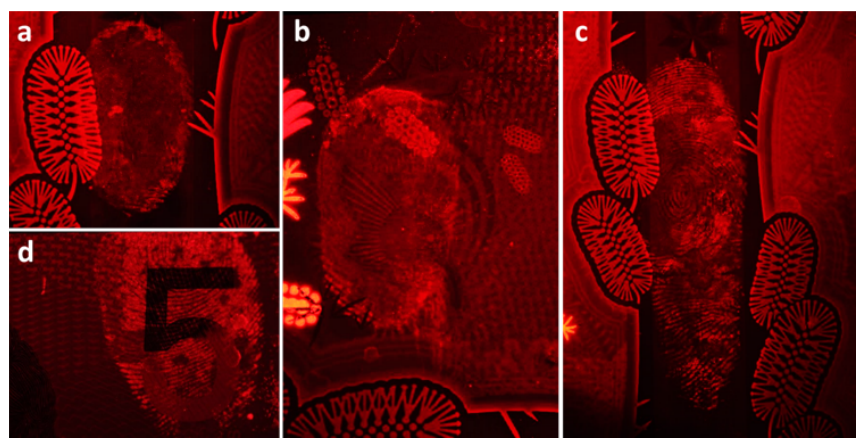

Figure 4. Treated (shaken at $20 \mathrm{rpm}$ for $10 \mathrm{~min}$ in CIS-ZS QDs solution) 8-days-old latent fingermarks on Australian polymer banknote. The marks were illuminated at $350 \mathrm{~nm}$ and pictured through the deep red filter. See Table S1 for the photography conditions.

\subsection{Conclusions}

In conclusion, a safe innovative and straightforward procedure was developed for preparation and application of an aqueous working solution containing surface modified CIS-ZS core-shell QDs for visualisation of latent fingermarks. The surface modification consists of a surfactant coating which allows dispersion of the QDs in aqueous solution, as well as providing the capability of being attracted to the latent fingermark residue. Latent fingermarks on various non-porous substrates were rapidly developed with high levels of ridge detail being observed. Being luminescent at deep red to NIR wavelength made it possible to eliminate the problem of dark, multi-coloured and multi-patterned backgrounds. However, issues remain in application to natural fingermarks on some challenging substrates such as polymer banknotes and cans. Further studies with a wider range of donors and substrates are required, as well as optimisation of the technique, including factors such as additional surface modification of the QDs, pH adjustment of the working solution, working solution stability/shelf-life and combination with other methods (such as cyanoacrylate fuming).

\section{Acknowledgements}

The authors thank all the fingermark donors for their cooperation. We thank the Reserve Bank of Australia (RBA) for supply of the banknotes in this study and the associated permission to damage the notes in the course of the research. This research has been conducted in accordance with the Curtin University human research ethics committee (approval number: HRE2016-0252). S.S. and R.B. were supported by an Australian Postgraduate Award (APA) and Research Training Scheme (RTS) awarded from Curtin University. G.J. acknowledges ARC for funding support through the DECRA scheme (Project ID: DE160100589). N.N.D. and O.A. gratefully acknowledge funding from the Leverhulme Trust. There are no conflicts to declare.
Keywords: Latent fingermarks $\bullet$ quantum dots $\bullet$ photoluminescence $\bullet$ forensic science

\section{References}

1. Moret, S., A. Bécue, and C. Champod, Nanoparticles for fingermark detection: An insight into the reaction mechanism. Nanotechnology, 2014. 25: p. 425502.

2. Dilag, J., H. J. Kobus, and A. V. Ellis, Nanotechnology as a New Tool for Fingermark Detection: A Review. Current Nanoscience, 2011. 7(2): p. 153-159.

3. Bécue, A. and A. Cantú, Fingermark Detection Using Nanoparticles, in Lee and Gaensslen's Advances in Fingerprint Technology, Third Edition. 2012, CRC Press. p. 307-380.

4. Choi, M.J., et al., Metal-containing nanoparticles and nanostructured particles in fingermark detection. Forensic Science International, 2008. 179(2): p. 87-97.

5. Hai, J., et al., Reversible Response of Luminescent Terbium(III)Nanocellulose Hydrogels to Anions for Latent Fingerprint Detection and Encryption. Angewandte Chemie International Edition, 2018. 57(23): p. 6786-6790.

6. Menzel, E., et al., Photoluminescent CdS/Dendrimer Nanocomposites for Fingerprint Detection. Journal of Forensic Sciences, 2000. 45(4): p. 770-773.

7. Jia, G., et al., Couples of colloidal semiconductor nanorods formed by self-limited assembly. Nature Materials, 2014. 13: p. 301.

8. Algarra, M., et al., Fingerprint detection and using intercalated CdSe nanoparticles on non-porous surfaces. Analytica Chimica Acta, 2014. 812: p. 228-235.

9. Liu, J., et al., Water-soluble multicolored fluorescent CdTe quantum dots: Synthesis and application for fingerprint developing. Journal of Colloid and Interface Science, 2010. 342(2): p. 278-282.

10. Yu, X., et al., Application of mercaptosuccinic acid capped CdTe quantum dots for latent fingermark development. Forensic Science International, 2013. 231(1): p. 125-130.

11. Bottrill, M. and M. Green, Some aspects of quantum dot toxicity. Chemical Communications, 2011. 47(25): p. 7039-7050.

12. Wang, Y.F., et al., Application of CdSe nanoparticle suspension for developing latent fingermarks on the sticky side of adhesives. Forensic Science International, 2009. 185(1): p. 9699.

13. Zhao, Y.-B., et al., New luminescent nanoparticles based on carbon dots $/ \mathrm{SiO}_{2}$ for the detection of latent fingermarks. Analytical Methods, 2017. 9(33): p. 4770-4775.

14. Moret, S., A. Bécue, and C. Champod, Cadmium-free quantum dots in aqueous solution: Potential for fingermark detection, synthesis and an application to the detection of fingermarks in blood on non-porous surfaces. Forensic Science International, 2013. 224(1): p. 101-110.

15. $\mathrm{Xu}, \mathrm{C}$., et al., Fast Imaging of Eccrine Latent Fingerprints with Nontoxic Mn-Doped ZnS QDs. Analytical Chemistry, 2014. 86(7): p. 3279-3283.

16. Hazarika, P. and D. Russell, Advances in Fingerprint Analysis. Angewandte Chemie (International ed. in English), 2012. 51: p. 3524-31.

17. Sharma, V., et al., Potential of $\mathrm{Sr}_{4} \mathrm{Al}_{1}{ }_{4} \mathrm{O}_{25}: \mathrm{Eu}^{2+}, \mathrm{Dy}{ }^{3+}$ inorganic oxide-based nanophosphor in latent fingermark detection. Journal of Materials Science, 2013. 49: p. 1-10.

18. King, R.S.P., P.M. Hallett, and D. Foster, Seeing into the infrared: A novel IR fluorescent fingerprint powder. Forensic Science International, 2015. 249: p. e21-e26.

19. Chadwick, S., et al., Styryl dye coated metal oxide powders for the detection of latent fingermarks on non-porous surfaces. Forensic Science International, 2012. 219(1-3): p. 208-214.

20. Maynard, P., et al., Near infrared imaging for the improved detection of fingermarks on difficult surfaces. Australian Journal of Forensic Sciences, 2009. 41(1): p. 43-62.

21. Xie, R., M. Rutherford, and X. Peng, Formation of High-Quality I-III-VI Semiconductor Nanocrystals by Tuning Relative 
Reactivity of Cationic Precursors. Journal of the American Chemical Society, 2009. 131(15): p. 5691-5697.

22. Li, L., et al., Efficient Synthesis of Highly Luminescent Copper Indium Sulfide-Based Core/Shell Nanocrystals with Surprisingly Long-Lived Emission. Journal of the American Chemical Society, 2011. 133(5): p. 1176-1179.

23. Zhong, H., et al., Noninjection Gram-Scale Synthesis of Monodisperse Pyramidal CulnS2 Nanocrystals and Their SizeDependent Properties. ACS Nano, 2010. 4(9): p. 5253-5262.

24. Panthani, M.G., et al., Synthesis of CulnS 2 , CulnSe 2 , and $\mathrm{Cu}$ (InxGa1-x)Se $\mathrm{S}_{2}$ (CIGS) Nanocrystal "Inks" for Printable Photovoltaics. Journal of the American Chemical Society, 2008. 130(49): p. 16770-16777.

25. Zhang, W. and X. Zhong, Facile Synthesis of ZnS-CulnS ${ }_{2}$-Alloyed Nanocrystals for a Color-Tunable Fluorchrome and Photocatalyst. Inorganic Chemistry, 2011. 50(9): p. 4065-4072.

26. Xiong, W.-W., et al., Aqueous Synthesis of Color-Tunable CuInS $2 / Z n S$ Nanocrystals for the Detection of Human Interleukin 6. ACS Applied Materials \& Interfaces, 2013. 5(16): p. 82108216.

27. Li, L., et al., Highly Luminescent CulnS $2 / Z n S$ Core/Shell Nanocrystals: Cadmium-Free Quantum Dots for In Vivo Imaging. Chemistry of Materials, 2009. 21(12): p. 2422-2429.

28. Pons, T., et al., Cadmium-Free CuInS $2 / Z n S$ Quantum Dots for Sentinel Lymph Node Imaging with Reduced Toxicity. ACS Nano, 2010. 4(5): p. 2531-2538.

29. Deng, D., et al., High-quality CulnS $2 / Z n S$ quantum dots for in vitro and in vivo bioimaging. Chemistry of Materials, 2012. 24(15): p. 3029-3037.

30. Mohan, S., et al., Green synthesis of yellow emitting PMMA$\mathrm{CdSe} / \mathrm{ZnS}$ quantum dots nanophosphors. Materials Science in Semiconductor Processing, 2015. 39: p. 587-595.

31. Yarema, O., M. Yarema, and V. Wood, Tuning the Composition of Multicomponent Semiconductor Nanocrystals: The Case of IIII-VI Materials. Chemistry of Materials, 2018. 30(5): p. 14461461.
32. Becue, A., et al., Use of quantum dots in aqueous solution to detect blood fingermarks on non-porous surfaces. Forensic Science International, 2009. 191(1): p. 36-41.

33. Chen, Y., et al., Green and Facile Synthesis of Water-Soluble CuIn-S/ZnS Core/Shell Quantum Dots. Inorganic Chemistry, 2013. 52(14): p. 7819-7821.

34. Peng, X., et al., Epitaxial Growth of Highly Luminescent CdSe/CdS Core/Shell Nanocrystals with Photostability and Electronic Accessibility. Journal of the American Chemical Society, 1997. 119(30): p. 7019-7029.

35. Pan, Z., et al., High-Efficiency "Green" Quantum Dot Solar Cells. Journal of the American Chemical Society, 2014. 136(25): p. 9203-9210.

36. Liu, S., et al., One-pot synthesis of ternary CuInS $S_{2}$ quantum dots with near-infrared fluorescence in aqueous solution. RSC Advances, 2012. 2(3): p. 819-825.

37. Raevskaya, A., et al., Non-stoichiometricCu-In-S@ZnS nanoparticles produced in aqueous solutions as light harvesters for liquid-junction photoelectrochemical solar cells. RSC Advances, 2016. 6(102): p. 100145-100157.

38. Zhang, B., et al., The composition effect on the optical properties of aqueous synthesized $\mathrm{Cu}-\mathrm{In}-\mathrm{S}$ and $\mathrm{Zn}-\mathrm{Cu}-\mathrm{In}-\mathrm{S}$ quantum dot nanocrystals. Physical Chemistry Chemical Physics, 2015. 17(38): p. 25133-25141.

39. Bao, $\mathrm{H}$., et al., Enhancement Effect of Illumination on the Photoluminescence of Water-Soluble CdTe Nanocrystals: Toward Highly Fluorescent CdTe/CdS Core-Shell Structure. Chemistry of Materials, 2004. 16(20): p. 3853-3859.

40. Prescott, L.F., et al., Treatment of paracetamol (acetaminophen) poisoning with $n$-acetylcysteine. The Lancet, 1977. 310(8035): p. 432-434.

41. IFRG, Guidelines for the Assessment of Fingermark Detection Techniques. Journal of Forensic Identification, 2014. 64(2): p. 174-200. 

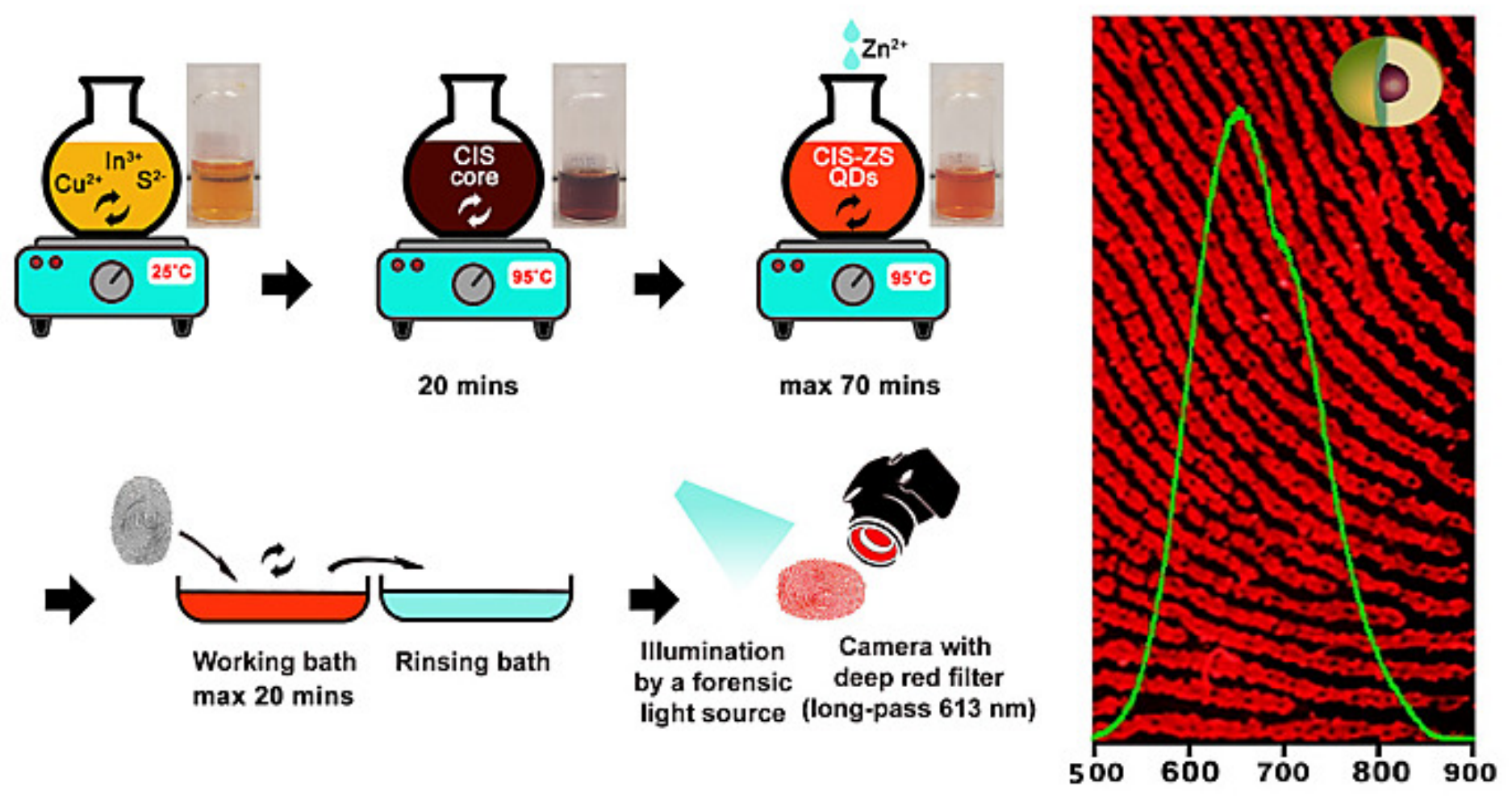


\section{Supplementary Information: Luminescence Detection of Latent Fingermarks}

\section{on Non-porous surfaces with Heavy-Metal-Free Quantum Dots}

Sorour Shahbazi, ${ }^{a}$ Rhiannon Boseley, ${ }^{a}$ Braden Grant, ${ }^{a}$ Dechao Chen, ${ }^{\text {a }}$ Thomas Becker, ${ }^{\text {a }}$ Oluwasesan Adegoke, ${ }^{b}$

Niamh NicDaéid, ${ }^{\text {b }}$ Guohua Jia, ${ }^{* a}$ Simon W. Lewis*a

${ }^{a}$ Curtin Institute of Functional Molecules and Interfaces, School of Molecular and Life Sciences, Curtin University, GPO Box U1987, Perth,

WA, 6845.

${ }^{b}$ Leverhulme Research Centre for Forensic Science, School of Science and Engineering, University of Dundee, Scotland, UK.

*Email: s.lewis@curtin.edu.au; guohua.jia@curtin.edu.au

Table S1. Photography specifications of the photos taken from the latent fingermarks

\begin{tabular}{|c|c|c|c|c|c|}
\hline Figure No. & F-stop & $\begin{array}{c}\text { Exposure time } \\
\text { (sec) }\end{array}$ & Figure No. & F-stop & $\begin{array}{c}\text { Exposure time } \\
\text { (sec) }\end{array}$ \\
\hline $\mathbf{2 a}$ & $\mathrm{f} / 3.2$ & 5 & $\mathbf{S 2}$ & $\mathrm{f} / 13$ & 5 \\
\hline $\mathbf{2 b}$ & $\mathrm{f} / 3.2$ & 10 & S5 & $\mathrm{f} / 3.2$ & 6 \\
\hline $\mathbf{2 c}$ & $\mathrm{f} / 3.2$ & 20 & S6a & $\mathrm{f} / 3.2$ & 5 \\
\hline $\mathbf{2 d}$ & $\mathrm{f} / 3.2$ & 10 & S6b & $\mathrm{f} / 3.2$ & 10 \\
\hline $\mathbf{2 e}$ & $\mathrm{f} / 3.2$ & 2 & S6c-e & $\mathrm{f} / 3.2$ & 30 \\
\hline $\mathbf{2 f}$ & $\mathrm{f} / 3.2$ & 1 & S6f & $\mathrm{f} / 3.2$ & 20 \\
\hline $\mathbf{2 g}$ & $\mathrm{f} / 4$ & 15 & S6g-r & $\mathrm{f} / 4$ & 15 \\
\hline $\mathbf{3 a}, \mathbf{c}, \mathbf{e}$ & $\mathrm{f} / 11$ & $3 / 5$ & S7a & $\mathrm{f} / 3$ & 8 \\
\hline $\mathbf{3 b}$ & $\mathrm{f} / 3.2$ & 2 & S7b & $\mathrm{f} / 3.2$ & 1 \\
\hline $\mathbf{3 d}$ & $\mathrm{f} / 3.2$ & $8 / 5$ & S8a-b & $\mathrm{f} / 3.5$ & $5 / 2$ \\
\hline $\mathbf{3 f}$ & $\mathrm{f} / 3.2$ & 1 & S8c & $\mathrm{f} / 3.5$ & 4 \\
\hline $\mathbf{3 g}$ & $\mathrm{f} / 3.2$ & $1 / 8$ & S8d & $\mathrm{f} / 3.5$ & 6 \\
\hline
\end{tabular}
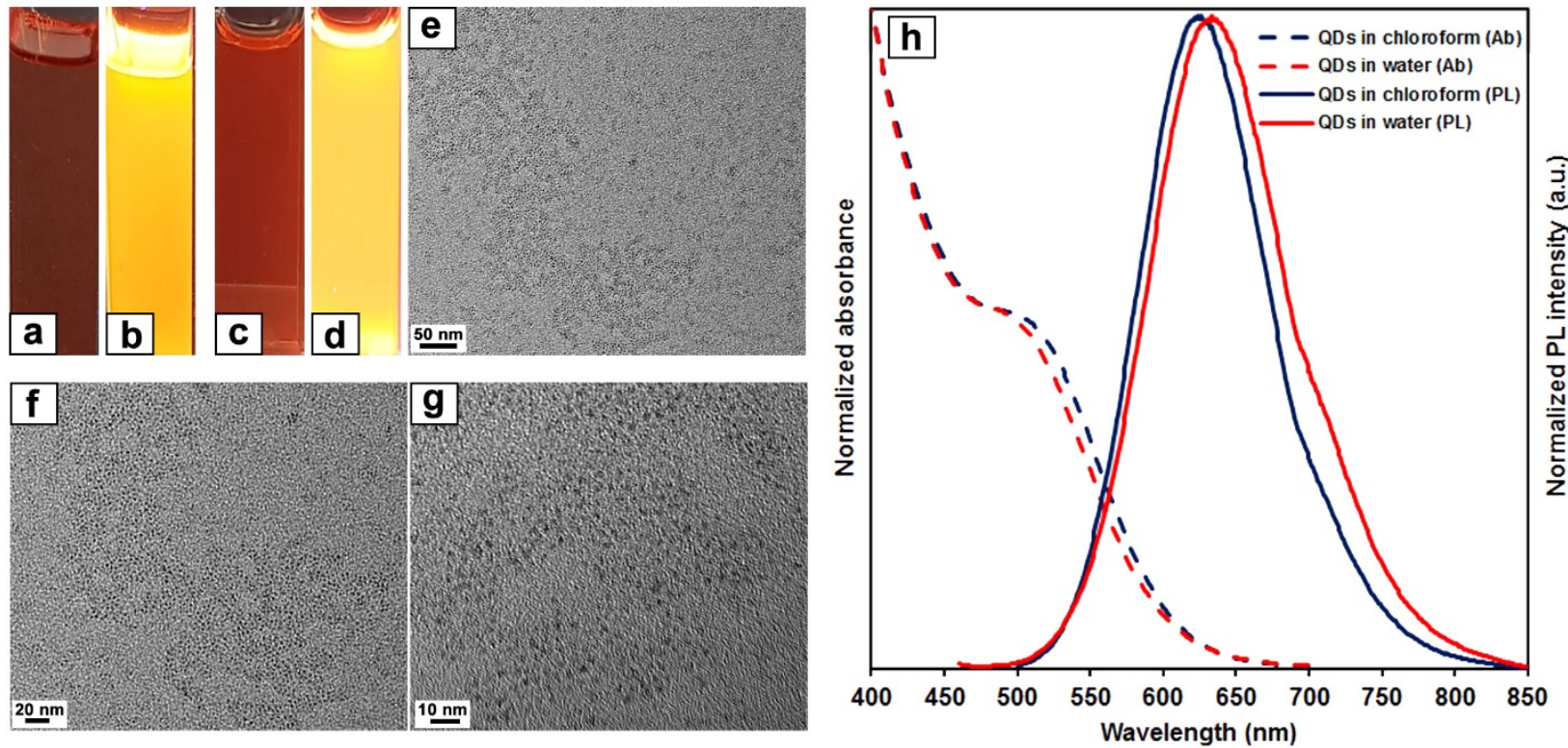

Figure S1. ( $a-d)$ CIS-ZS QDs dispersed in ( $a$ and b) chloroform and ( $c$ and $d$ ) in water and photographed under (a and c) no illumination and ( $b$ and d) $350 \mathrm{~nm}$ illumination. (e-g) TEM images of the CIS-ZS QDs in different magnifications. (h) PL (excited at $450 \mathrm{~nm}$ ) and Ab spectra of the QDs solutions. 


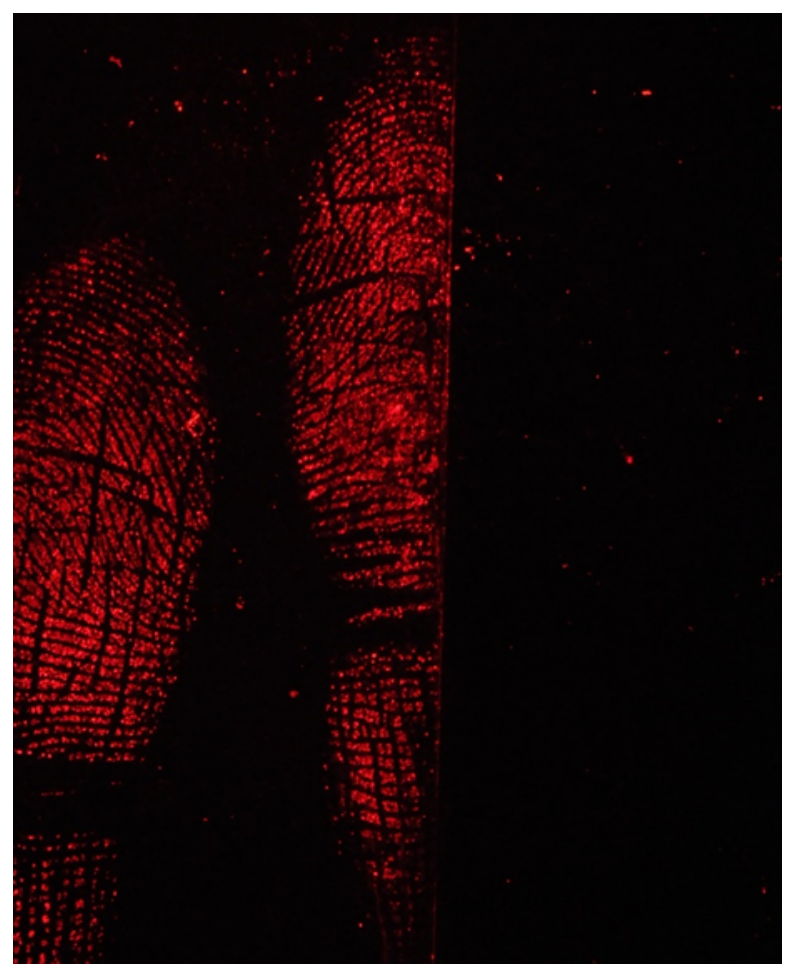

Figure S2. (a) Untreated (right side) and CIS-ZS QDs treated (left side) fresh charged split latent fingermarks (one female donor) on glass slides. The mark was illuminated at $350 \mathrm{~nm}$ and pictured through the deep red filter. See Table S1 for the photography conditions. 

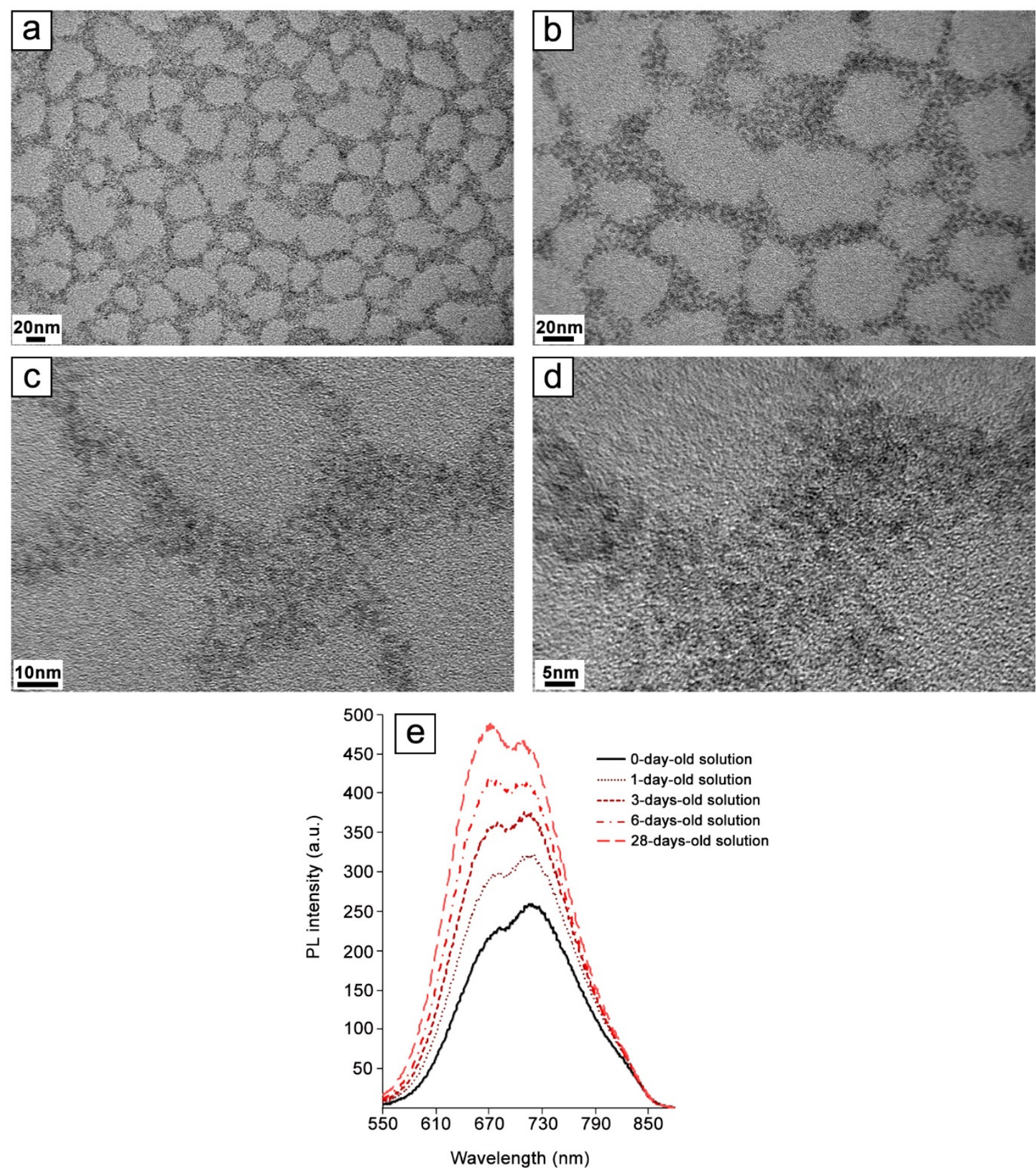

Figure S3. (a-d) TEM images of the CIS-ZS core-shell QDs synthesised in water in different magnifications. (e) PL spectra of fresh and old CIS-ZS QDs solutions. (f-g) CIS-ZS QDs solutions (f) before and $(g)$ after addition of MPA. Inset in (f) is the precipitated $Q D$ s solution before the addition of MPA. 

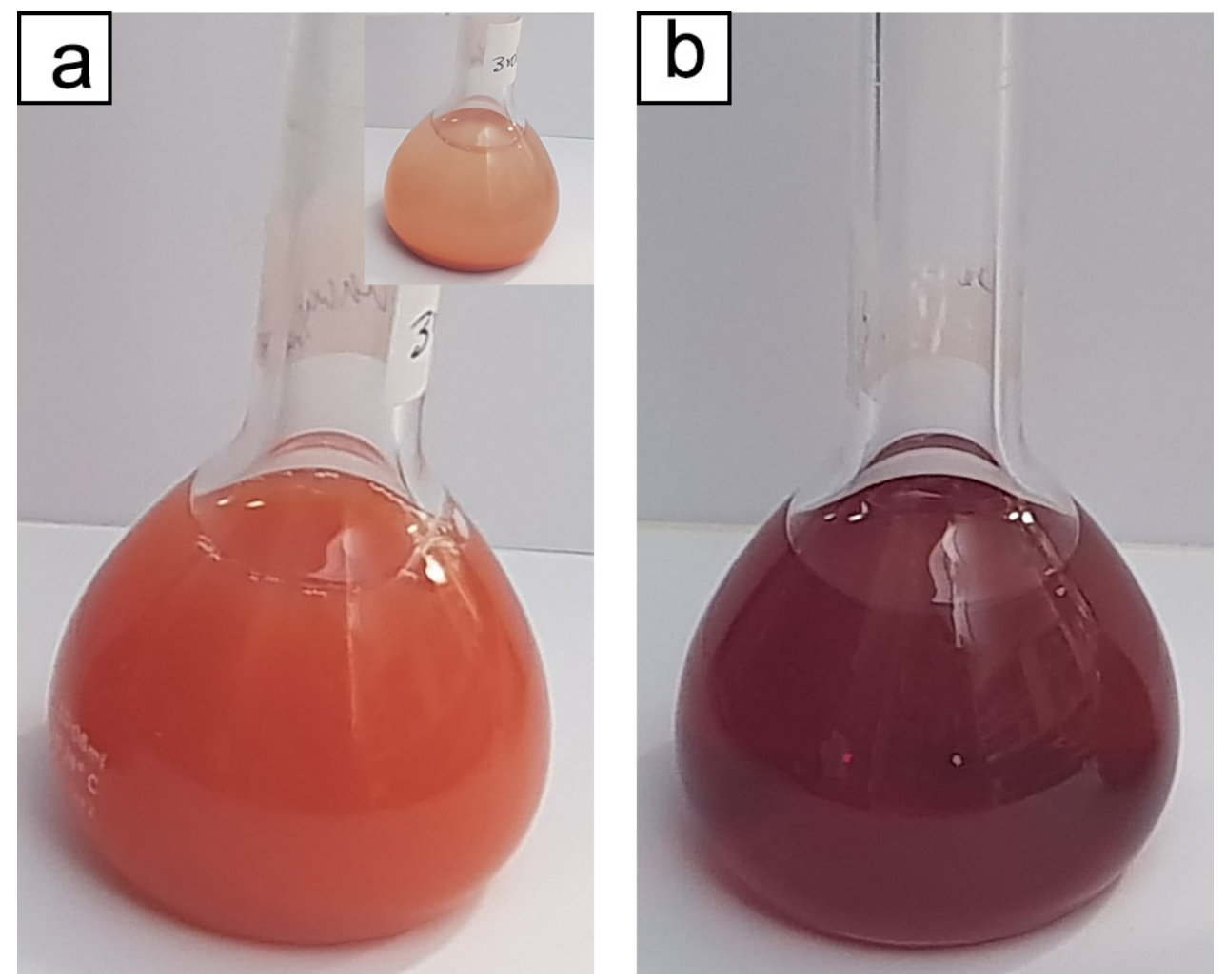

Figure S4. CIS-ZS QDs solutions (a) before and (b) after addition of MPA. Inset in (a) is the precipitated QDs solution before the addition of MPA.
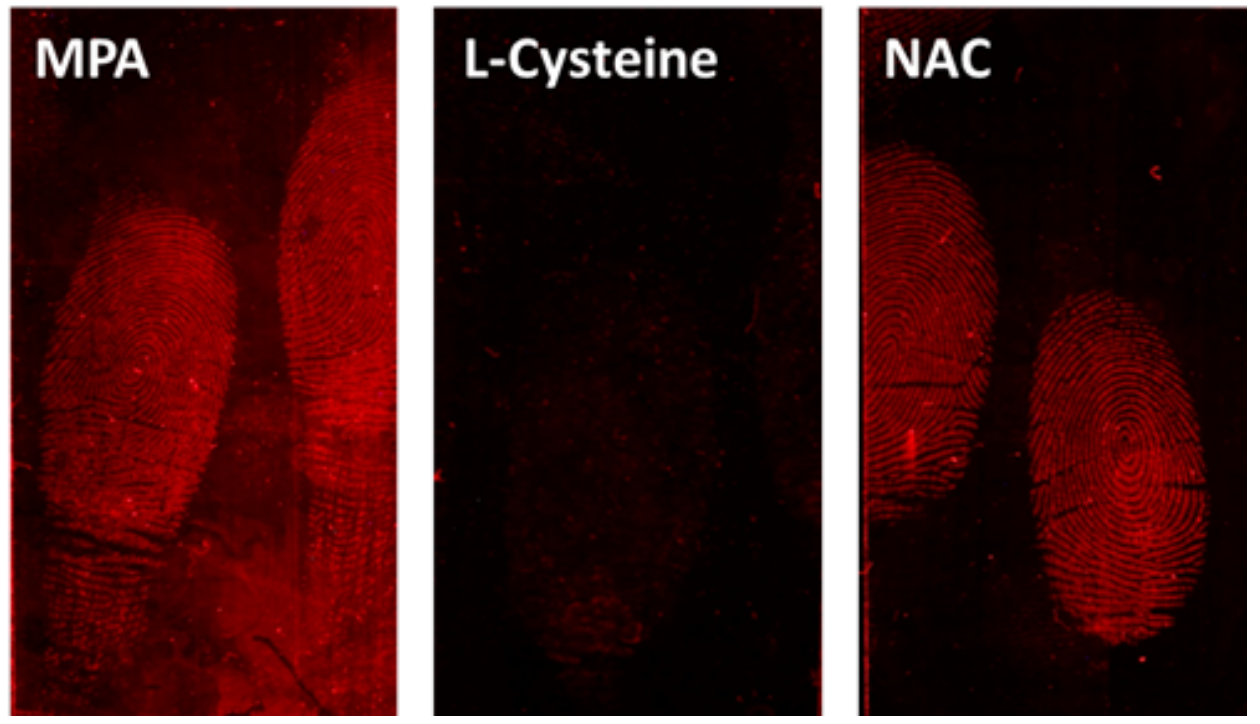

Figure S5 - Developed natural latent fingermark (one female donor) deposited on a transparent sticky tape. Developed with MPA-coated CIS-ZS QD's (left), L-cysteine-coated CIS-ZS QD's (middle), NAC-coated CIS-ZS QD's (right) solutions. The marks were illuminated at $350 \mathrm{~nm}$ and pictured through the deep red filter. 


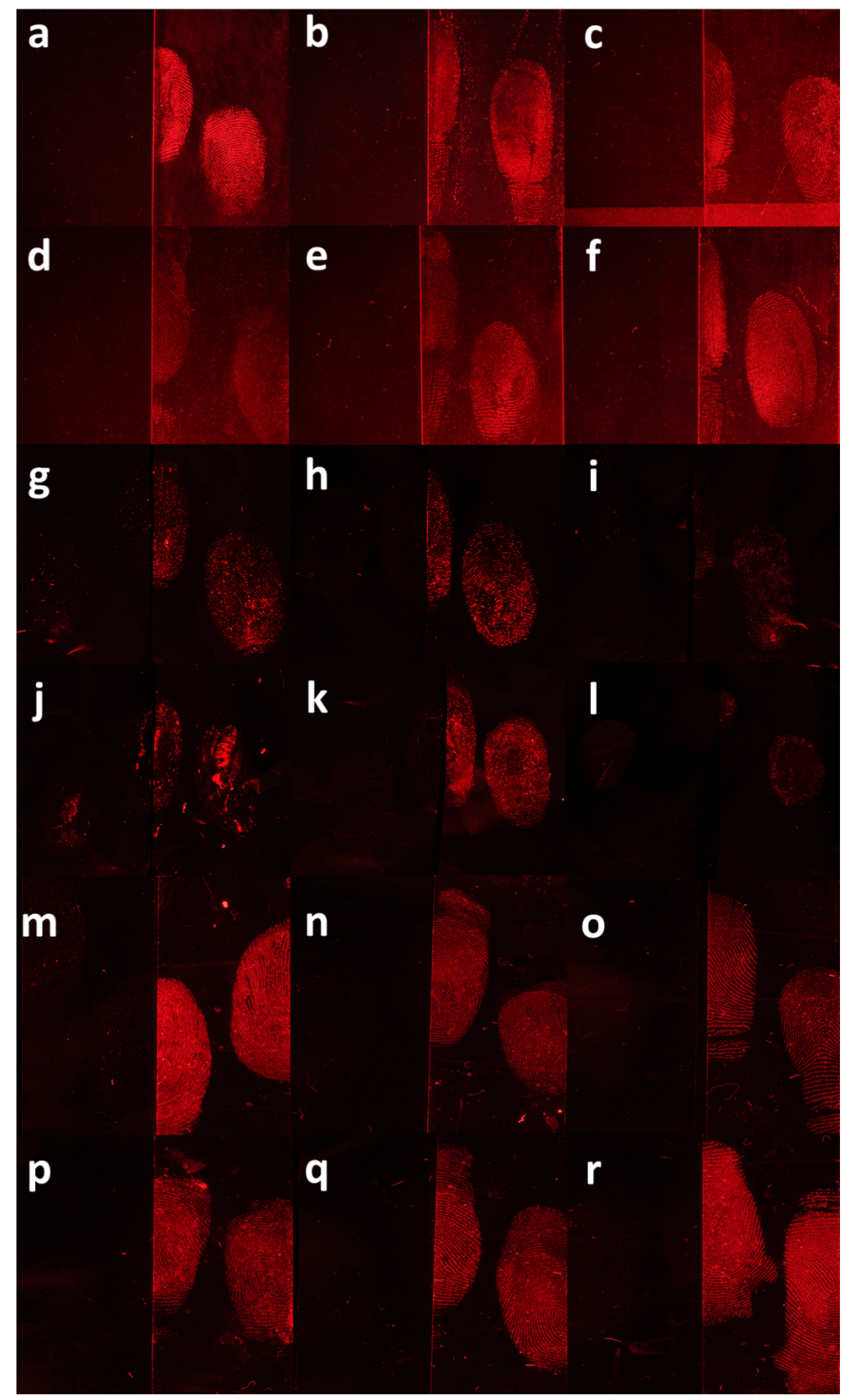

Figure S6. Untreated (left side) and treated (right side) natural split latent fingermarks (a-f) 1 day old on glass slides (g-l) $d$ ) 2 days old on aluminium foil (m-r) 3 days old on transparent adhesive tape, from a range of donors. The marks were illuminated at $350 \mathrm{~nm}$ and pictured through the deep red filter. See Table S1 for the photography conditions. 
